\title{
EchoGéo
}

25 | 2013

Moyen-Orient : espaces et passeurs du changement

\section{Espaces vécus et imaginés des rencontres amoureuses aux Émirats arabes unis}

Laure Assaf

\section{OpenEdition}

1 Journals

Édition électronique

URL : https://journals.openedition.org/echogeo/13538

DOI : $10.4000 /$ echogeo. 13538

ISSN : 1963-1197

Éditeur

Pôle de recherche pour l'organisation et la diffusion de l'information géographique (CNRS UMR 8586)

Référence électronique

Laure Assaf, "Espaces vécus et imaginés des rencontres amoureuses aux Émirats arabes unis », EchoGéo [En ligne], 25 | 2013, mis en ligne le 10 octobre 2013, consulté le 10 août 2021. URL : http:// journals.openedition.org/echogeo/13538; DOI : https://doi.org/10.4000/echogeo.13538

Ce document a été généré automatiquement le 10 août 2021.

EchoGéo est mis à disposition selon les termes de la licence Creative Commons Attribution - Pas d'Utilisation Commerciale - Pas de Modification 4.0 International (CC BY-NC-ND) 


\title{
Espaces vécus et imaginés des rencontres amoureuses aux Émirats arabes unis
}

\author{
Laure Assaf
}

\section{Introduction}

1 Mohammed est un Émirien de vingt-six ans. Mince, aux cheveux noirs et bouclés, il arrive légèrement en retard au rendez-vous que nous nous sommes fixé dans un café, sur la corniche d'Abou Dhabi. Il porte une dishdasha blanche (longue tunique considérée comme l'habit national émirien pour les hommes), et la ghutra (foulard carré) nouée sur la tête, à la manière des jeunes hommes. Il évoque avec humour son mariage raté et son récent divorce - une semaine après la cérémonie - qu'il regrette peu : il avait accepté par respect pour ses parents, qui ont arrangé le mariage, mais les rapports étaient conflictuels avec sa future épouse durant les trois mois des fiançailles. Et puis il est jeune, il a envie de s'amuser, d'avoir des petites amies. Ses parents n'y voient pas d'objection, mais à Abou Dhabi, si la fille est « locale » (d'une famille émirienne), il faut toujours se voir en secret - il a entendu des histoires de couples non mariés arrêtés par la police. Et si les policiers décident d'appeler les parents de la fille, les problèmes commencent. Avant, tous les couples se rencontraient sur la plage publique de Ra's AlAkhdâr, derrière Emirates Palace. Depuis qu'elle a fermé, il reste les parkings : celui de l'hôtel Intercontinental où personne ne va jamais, ou bien le grand parking toujours vide dans Officers' Club. Il y a aussi le dernier étage du parking d'Al-Wahda Mall ${ }^{1}$ : personne ne regarde les caméras de sécurité, et les couples peuvent y faire ce qu'ils veulent - à condition de rester dans la voiture aux vitres teintées, bien sûr. Le plus simple, c'est encore d'aller à Dubaï, ou de prendre son bateau personnel et de s'arrêter sur l'une des nombreuses petites îles qui entourent Abu Dhabi.

2 À l'image du récit de Mohammed, les relations amoureuses racontées par les jeunes adultes dans les Émirats arabes unis, qu'elles soient vécues ou fictives, prennent 
souvent une dimension spatiale importante. Cela tient en partie au fait que l'espace récent des villes émiriennes cristallise des normes sociales renforcées par la cohabitation de populations variées. Dans des villes qui ont été caractérisées par leurs constantes transformations et leurs projets architecturaux spectaculaires (Elsheshtawy, 2010 ; Kanna, 2011), l'espace urbain est en effet à l'image des changements rapides qui ont modifié la société émirienne depuis la découverte du pétrole ${ }^{2}$ (Davidson, 2009 ; Heard-Bey, 2005). Mais si Abou Dhabi ou Dubaï sont des objets de fascination, voire de fantasmes (Lavergne, 2005), pour les touristes comme pour une partie des migrants qui viennent s'y installer, pour les jeunes Émiriens et expatriés arabes qui ont grandi dans ces villes l'espace urbain est chargé de contraintes autant que de possibilités d'émancipation.

3 Comment caractériser la "dimension spatiale des normes sociales» (Banos, 2009) dans les villes émiriennes, et comment rendre compte de ces moments où les pratiques détournent les lieux, ou les (ré)inventent, pour mieux contourner les règles ? L'enquête ethnographique menée auprès de jeunes Émiriens et expatriés arabes suggère que les rencontres amoureuses des jeunes adultes dans les Émirats «traversent » ces espaces sans nécessairement transgresser ${ }^{3}$. A travers l'examen des espaces vécus et imaginés des rencontres amoureuses aux Émirats arabes unis, cet article interroge la manière dont l'espace peut être mobilisé par les jeunes Émiriens et expatriés arabes pour s'émanciper dans une certaine mesure des normes sociales - tout en questionnant l'étendue et les limites de cette émancipation. Il se fonde sur une ethnographie menée auprès de jeunes adultes (de 20 à 30 ans environ), Émiriens et expatriés arabes ayant grandi aux Émirats arabes unis, dans le cadre d'une thèse de doctorat portant de manière plus générale sur les sociabilités urbaines de ces jeunesses arabes à Abou Dhabi. Le sujet des relations amoureuses a donc été la plupart du temps abordé indirectement, à partir de la question du mariage ou des relations entre les genres. Il a toujours été soulevé par mes interlocuteurs eux-mêmes, parfois dans le cadre de la confidence (en révélant une relation qu'ils entretenaient), sous la forme d'histoires rapportées destinées à m'instruire sur les pratiques des autres et auxquelles ils donnaient quelquefois une connotation morale négative, ou bien encore entre eux, au sein de conversations en groupe ${ }^{4}$. Aux récits individuels s'ajoutent les pratiques souvent collectives de flirt et de drague.

L'examen de l'accès aux espaces publics urbains en fonction des rapports de genre et de nationalité permettra d'abord de faire émerger une typologie des lieux de rencontre dans la ville émirienne et des normes qui leur sont associées - chaque lieu ayant une valeur sociale et une réputation, qui dépendent en partie des catégories de population qui les fréquentent. On mettra ainsi à jour une certaine tension entre les contraintes pesant sur la sociabilité des jeunes adultes et les interactions offertes par la ville cosmopolite. Cette tension donne lieu à des stratégies de diversion jouant sur les possibilités des lieux, mais aussi sur la frontière entre le transgressif et le respectable, qui questionnent en retour la définition de l'espace urbain des villes du Golfe. A ces espaces vécus des rencontres amoureuses s'ajoute la recherche de l'ailleurs, à travers les espaces imaginés dans les productions culturelles de ces jeunes adultes, blogs et courts-métrages, mais aussi à travers internet et l'usage de réseaux sociaux en ligne. 


\section{Genre, nationalité et accès à la ville}

$5 \quad$ S'il existe de grandes variations dans les catégories du permis et de l'interdit suivant le genre, le contexte familial, l'âge ou le statut social, la plupart des jeunes adultes que j'ai rencontrés identifiaient tout type de relation intime avec une personne du sexe opposé comme de l'ordre de l'interdit ${ }^{5}$ - qu'ils soient ou non d'accord avec ce constat. Cela comprend bien sûr les relations sexuelles, mais aussi inviter quelqu'un chez soi, ou simplement se retrouver ensemble dans certains lieux. En revanche, la mixité est souvent mieux tolérée et considérée comme plus légitime dans le cadre professionnel, voire éducatif (De Koning, 2009 ; Kreil, 2012) - même si, pour certaines jeunes femmes, elle peut constituer un obstacle dans l'accès à ces milieux.

6 Les normes portent donc sur des configurations sociales, sur des combinaisons de lieux, de personnes et de relations, plutôt que sur une seule de ces dimensions. Ainsi le caractère respectable ou non des lieux varie suivant les contextes et les individus, mais aussi selon les usages, le moment de la journée, ou le statut économique et social de ceux qui les fréquentent. A l'instar des personnes, en effet, les espaces urbains ont une réputation $\left(\operatorname{sum}^{\prime} a\right)$, fluctuante dans la mesure où elle dépend souvent de récits rapportés qui façonnent les représentations. Ainsi 'Alya, une étudiante émirienne de vingt-et-un ans, explique pourquoi sa famille lui interdit la fréquentation des hôtels ${ }^{6}$ : «Tu vois, un garçon, il peut faire [...] beaucoup de mauvaises choses, mais quand il s'assoit dans ces endroits [les hôtels], et qu'il voit une femme, il va présumer qu'elle est aussi mauvaise... A cause de l'endroit. Ce qui est complètement absurde bien sûr. [...] Mais c'est comme ça que ça marche». Shayma, une autre étudiante, ajoute: "Ils identifient le lieu en fonction des gens qui viennent dans ce lieu ».

7 Les lieux sont donc marqués par certains types de comportements, et la valeur sociale qui leur est ainsi attribuée affecte en retour ceux et surtout celles qui s'y rendent. Les hôtels, couramment associés à l'alcool (ce sont les seuls lieux pouvant vendre de l'alcool aux Émirats), aux boîtes de nuit et à la prostitution, sont des lieux à éviter, la présence y étant immédiatement suspecte. Le genre est un déterminant important dans l'accès aux espaces publics, et le souci de la réputation concerne d'abord les jeunes femmes ${ }^{7}$. Il porte moins sur les actes que sur la visibilité : l'essentiel pour ces jeunes femmes est de ne pas être vues au mauvais endroit par quelqu'un qui pourrait les reconnaître, ce qui signifie souvent un membre de la famille élargie (Le Renard, 2011a). Mais les lieux sont avant tout des cadres d'interaction, et il existe de nombreuses manières de jouer sur les autres composantes du contexte pour modifier la valeur sociale d'une situation. Ainsi, l'entretien avec 'Alya et Shayma s'est déroulé dans un hôtel... mais en matinée, dans les jardins, et dans le cadre de ma recherche universitaire. Leurs familles l'ignoraient et n'auraient sans doute pas donné leur accord, mais si elles étaient aperçues, je fournissais une raison légitime à leur présence ${ }^{8}$.

8 Si les jeunes hommes sont plus libres de leurs déplacements dans la ville et ont accès à la majorité des espaces publics, ils n'échappent pas pour autant à ces normes. Ainsi, lors de l'une de nos rencontres, Ahmad, un jeune Syrien de vingt-deux ans né à Abou Dhabi, me raconte une dispute avec ses parents qui a eu lieu la veille. Ces derniers ont exigé de consulter son répertoire téléphonique, et lui ont reproché le trop grand nombre de prénoms féminins parmi ses contacts. S'ils nous voyaient, ajoute Ahmad nous sommes en train de nous promener sur la corniche du bord de mer à Abou Dhabi - 
ils penseraient sans doute que je suis sa petite amie, et se mettraient à le surveiller de plus près. De nombreux auteurs ont réfléchi sur le caractère anormal de la situation ethnographique, et sur la nécessité pour l'ethnologue de négocier sa place auprès du groupe social qu'il étudie (Schwartz, 1993 ; Olivier de Sardan, 1995). Dans le cas de mon enquête à Abou Dhabi, la dimension spatiale de cette place a participé aux stratégies de mes interlocuteurs pour contourner certaines normes.

Cet accès différencié aux espaces publics en fonction du genre se trouve renforcé, dans les Émirats arabes unis, par la distinction entre nationaux et étrangers. En effet, la majorité de la population est constituée de migrants, venus majoritairement d'Asie du sud, du Moyen-Orient ou encore des pays occidentaux (Kapiszewski, 2001). Leur présence dans le pays est conditionnée à l'obtention d'un contrat de travail et à l'existence d'un garant ( $k a f i l)$, et la position et le salaire dépendent en partie de la nationalité ${ }^{9}$ (Longva, 1999). Les citoyens émiriens représentent environ $19 \%$ de la population totale à Abou Dhabi, et $11 \%$ à Dubaï. Malgré les barrières financières imposées au regroupement familial (l'exigence d'un revenu minimal sans cesse augmenté), de plus en plus de migrants font venir leurs familles - les familles des jeunes expatriés arabes que j'ai interrogés sont présentes dans le pays depuis souvent deux voire trois générations.

Si la population nationale bénéficie des privilèges matériels de la rente pétrolière ${ }^{10}$, elle perçoit cependant ces transformations sociales avec une certaine méfiance. Ainsi le déséquilibre démographique entre étrangers et nationaux est considéré comme une menace pour une culture et une identité nationales d'autant plus fragiles qu'elles sont de construction récente: les Émirats arabes unis ont été fondés en 1971. Les inquiétudes se cristallisent autour d'un certain nombre de problèmes, telle la question des mariages exogames, et en particulier des hommes émiriens épousant des femmes étrangères. Ce sujet revient régulièrement dans le débat public depuis au moins le milieu des années 1980 (Dresch, 2005; Bristol-Rhys, 2010). Plus récemment, la rhétorique de l'invasion culturelle a pris une dimension spatiale, à travers des conflits autour des normes de comportement et des codes vestimentaires dans les espaces publics ${ }^{11}$. La cause perçue de ces conflits est la coexistence, dans les villes émiriennes, de diverses nationalités et donc de normes sociales différentes, en particulier en ce qui concerne les relations entre les genres ou le degré d'intimité qu'il est possible de manifester dans l'espace public.

11 Les rapports entre les genres et les manières d'être dans l'espace public constituent ainsi des enjeux dans la différenciation entre nationaux et étrangers, dans les Émirats arabes unis comme dans le reste de la péninsule arabique ${ }^{12}$ (Le Renard, 2011b). Ces enjeux contribuent certainement à renforcer les normes de genre et les contraintes qui pèsent sur la sociabilité et les fréquentations des jeunes adultes. Les citoyens se distinguent en effet des autres populations dans les villes émiriennes par des régimes de visibilité spécifiques (Lussault, 2003), dont font partie la dishdasha et la 'abâya ${ }^{13}$, considérés comme les vêtements nationaux respectivement pour les hommes et les femmes (Khalaf, 2005). Aux normes sociales, familiales et religieuses qu'ils partagent avec les expatriés arabes s'ajoutent donc, pour les jeunes citoyens, la nécessité de se mettre en scène comme Émirien ou Émirienne dans la ville ${ }^{14}$. 


\section{Les relations amoureuses dans la ville : des parkings aux shopping malls}

12 Les normes régulant les relations entre les genres et les nationalités dans l'espace public des villes émiriennes mettent ainsi en jeu des questions et des débats transversaux à l'ensemble de la société. Dans ces conditions, les rencontres amoureuses avant le mariage apparaissent d'emblée comme problématiques, dans la mesure où elles remettent en question la ségrégation entre les genres dans l'espace public et les interdits portant sur les relations intimes. Les jeunes adultes mettent alors en œuvre une multiplicité de stratégies qui jouent sur les possibilités, les caractéristiques et les ressources spécifiques des lieux pour contourner les normes. L'analyse de ces appropriations nous permettra d'interroger la notion de transgression et ses significations aux Émirats arabes unis.

13 Le moyen-métrage " Shhh », réalisé en 2010 par deux étudiantes émiriennes, Hafsa alMutawwa' et Shammâ Abû Nawâs, aborde de front les questions du secret, de l'anonymat et de la transgression. Il met en scène les histoires croisées de quatre jeunes femmes dans la ville de Dubaï. Maithra, Mahra, Hind et Zânib sont de très bonnes amies que l'on voit ensemble faire du shopping, aller au salon de beauté pour une manucure ou encore faire une virée en voiture à Abou Dhabi le week-end, en se laissant draguer par de jeunes hommes à travers les fenêtres ouvertes. Mais le propos principal du film porte sur le secret que chacune d'entre elles dissimule aux autres. En développant les conséquences de chaque histoire, les réalisatrices rappellent les normes gouvernant les comportements amoureux des jeunes femmes autant qu'elles les subvertissent.

Hind sort le soir pour rejoindre quelqu'un dans une voiture - supposément une relation amoureuse, dont le spectateur découvre bientôt que l'autre protagoniste est une boya (contraction de l'anglais boy et de la marque du féminin en arabe). Le terme, propre à la péninsule arabique, désigne une jeune femme qui adopte un style visuel associé au masculin et subversif des modèles classiques de féminité (Le Renard, 2013 ; Al-Qasimi, $2010,2012)$ : cheveux courts et parfois teints, casquette, t-shirt large, pantalon baggy... Mais malgré son dégoût initial à l'idée d'être avec un homme, Hind finit par quitter son amie et accepter l'époux que ses parents ont choisi pour elle ${ }^{15}$.

15 Zânib, de son côté, se maquille : ongles manucurés, rouge à lèvres et chaussures à talons hauts contrastent avec son 'abâya. La difficulté à attirer l'attention des hommes malgré ses efforts pour se conformer aux canons de beauté et aux modes la poussent à contacter une entremetteuse dont elle a trouvé les coordonnées dans un magazine. Après lui avoir payé une importante somme d'argent, elle réussit à trouver un mari.

16 Maithra emmène son frère cadet au supermarché. Dans les rayonnages, un jeune homme la suit et l'observe. Il achète les bonbons qu'elle a refusés à son petit frère, et tend le sac à l'enfant en cadeau; à l'intérieur se trouve un morceau de papier sur lequel il a inscrit son numéro de téléphone. Elle l'appelle, ils se revoient au cinéma; mais elle met fin à leur relation lorsqu'elle découvre qu'il n'a pas l'intention de l'épouser.

17 Mahra sort d'une voiture, de nuit ; de la musique hip-hop s'échappe des véhicules garés tout autour. De jeunes hommes en dishdasha et d'autres en $t$-shirt et jeans discutent sur le parking; ils tournent la tête pour la regarder passer. On la retrouve assise dans un hall d'immeuble avec un groupe de jeunes gens, hommes et femmes. Ils font tourner une bouteille sur le sol, évoquant un jeu célèbre d'adolescents. Le lendemain matin, elle 
sort d'une chambre où elle a passé la nuit avec un jeune homme. Les amies de Mahra découvrent plus tard qu'elle fume et boit en cachette, et que de nombreuses rumeurs courent sur son compte. Elle est finalement exclue du groupe d'amies pour avoir tenté de contacter, par jalousie, le fiancé de Hind.

Le film, présenté dans divers festivals de cinéma à Dubaï et Abou Dhabi, a reçu des réactions ambivalentes, en raison des comportements transgressifs de ses héroïnes. Il peut être analysé à bien des niveaux. Le titre, "Shhh", évoque le silence censé recouvrir ces transgressions dans les Émirats arabes unis, tout en impliquant que les comportements montrés ne sont pas si rares. Il peut aussi être interprété différemment : le silence pesant sur les relations amoureuses, et de manière générale les interdits portant sur les relations entre les genres, conduiraient au secret et à des transgressions plus graves, représentées ici par l'homosexualité et des partenaires sexuels multiples ${ }^{16}$.

La ville - Dubaï, en l'occurrence - est l'une des dimensions de ces quatre histoires croisées, dans lesquelles elle apparaît par vignettes. Cet «archipel» (Gillot, 2005) d'espaces discontinus qui permettent dans une certaine mesure d'échapper aux normes rappelle le récit de Mohammed cité en introduction. Il désigne dans le paysage urbain des réseaux de lieux qui appartiennent à des catégories distinctes, associées à des types de relations différents (ou des étapes différentes au sein d'une relation donnée). Afin d'examiner ces liens, penchons-nous sur deux de ces catégories : d'une part, des lieux de consommation (les cafés, les centres commerciaux) dont le caractère global semble ouvrir la voie à une sociabilité mixte légitime. D'autre part, ceux que l'on pourrait qualifier, à la suite d'Andrew Gardner (2013), d'espaces interstitiels ou liminaires (les parkings, les terrains vagues, les plages...). Ces deux types d'espaces caractérisent l'urbanisme des villes du Golfe, qui juxtapose de manière discontinue des projets de développement modernes et spectaculaires, laissant entre eux des interstices que Gardner associe au compartimentage et à l'enclavement des différentes populations de ces villes (Gardner, 2013).

Les lieux de rencontre privilégiés des jeunes adultes à Abou Dhabi sont souvent des espaces à la fois très récents et dont la référence est globale: les shopping malls à l'américaine bâtis dans les années 2000, ou encore les coffee shops et les restaurants franchisés de chaînes internationales. Leur succès est dû, d'une part, à la rareté d'autres espaces publics légitimes dans la ville, mais également au fait que ces nouveaux espaces, parce qu'ils sont récents et importés, offrent « des disponibilités en termes d'usages publics » (Capron, 2000 ; Le Renard, 2011a). En ce sens ils sont ouverts à diverses modalités d'appropriation par les jeunesses arabes d'Abou Dhabi. Par ailleurs, le caractère global de ces espaces en fait à la fois des lieux respectables, et des lieux où une sociabilité amicale mixte en termes de genre est très légitime. Cela est dû à un certain nombre de facteurs : les prix pratiqués dans ces lieux excluent les couches les plus pauvres de la population, et notamment les travailleurs migrants, créant une exclusivité de classe (De Koning, 2009; Kreil, 2012). Il s'agit également de lieux très contrôlés, propres, surveillés, et dont le caractère public établit la possibilité du contrôle social (Assaf, 2013): dans les shopping malls, cette sociabilité amicale côtoie souvent une sociabilité plus familiale (cf. illustrations 1 et 2 ). 
Illustration 1- Sociabilités familiales et amicales à l'entrée de Marina Mall (Abou Dhabi), un soir de week-end

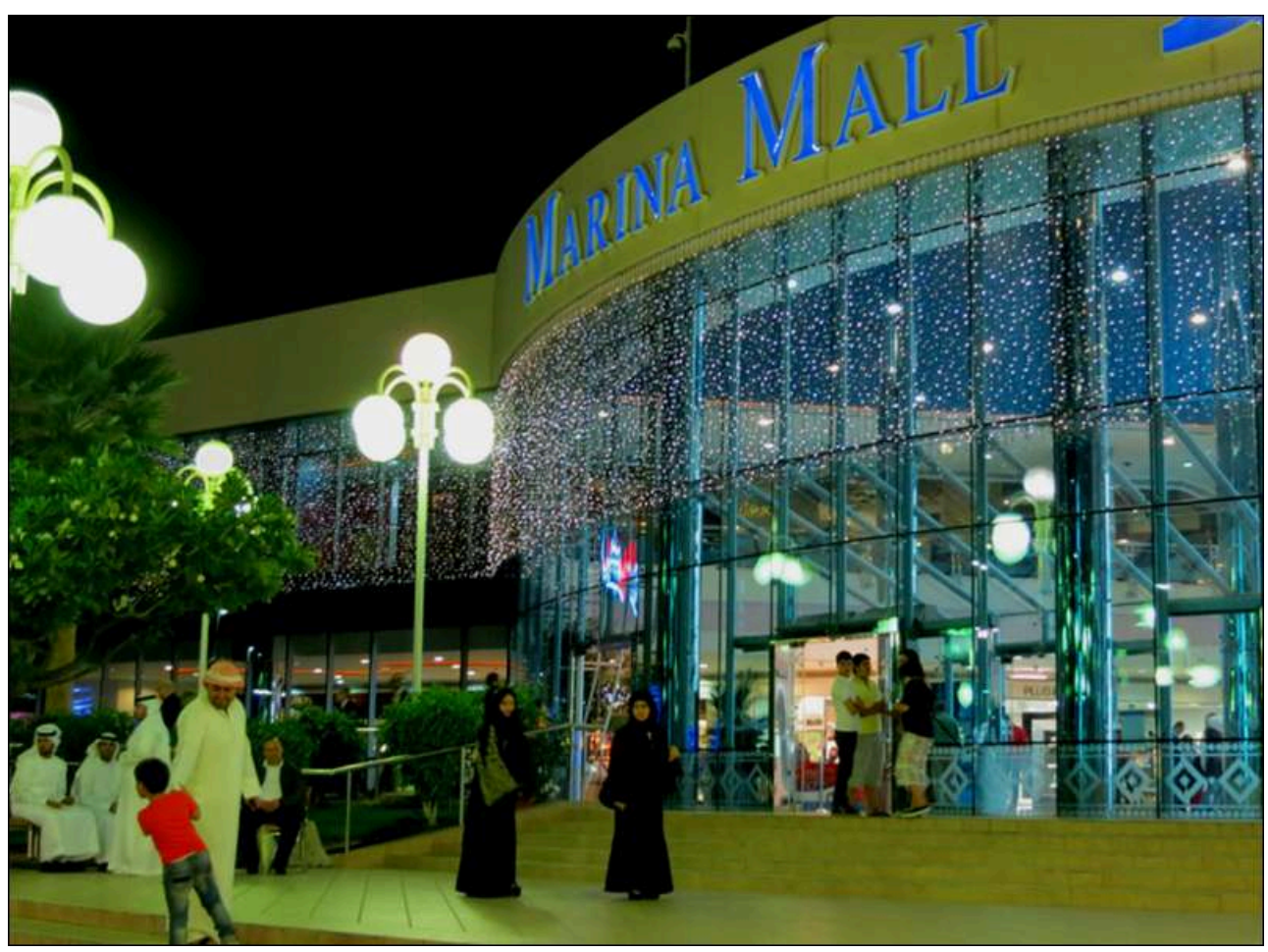

Illustration 2 - Café international à l'intérieur de Marina Mall (Abou Dhabi)

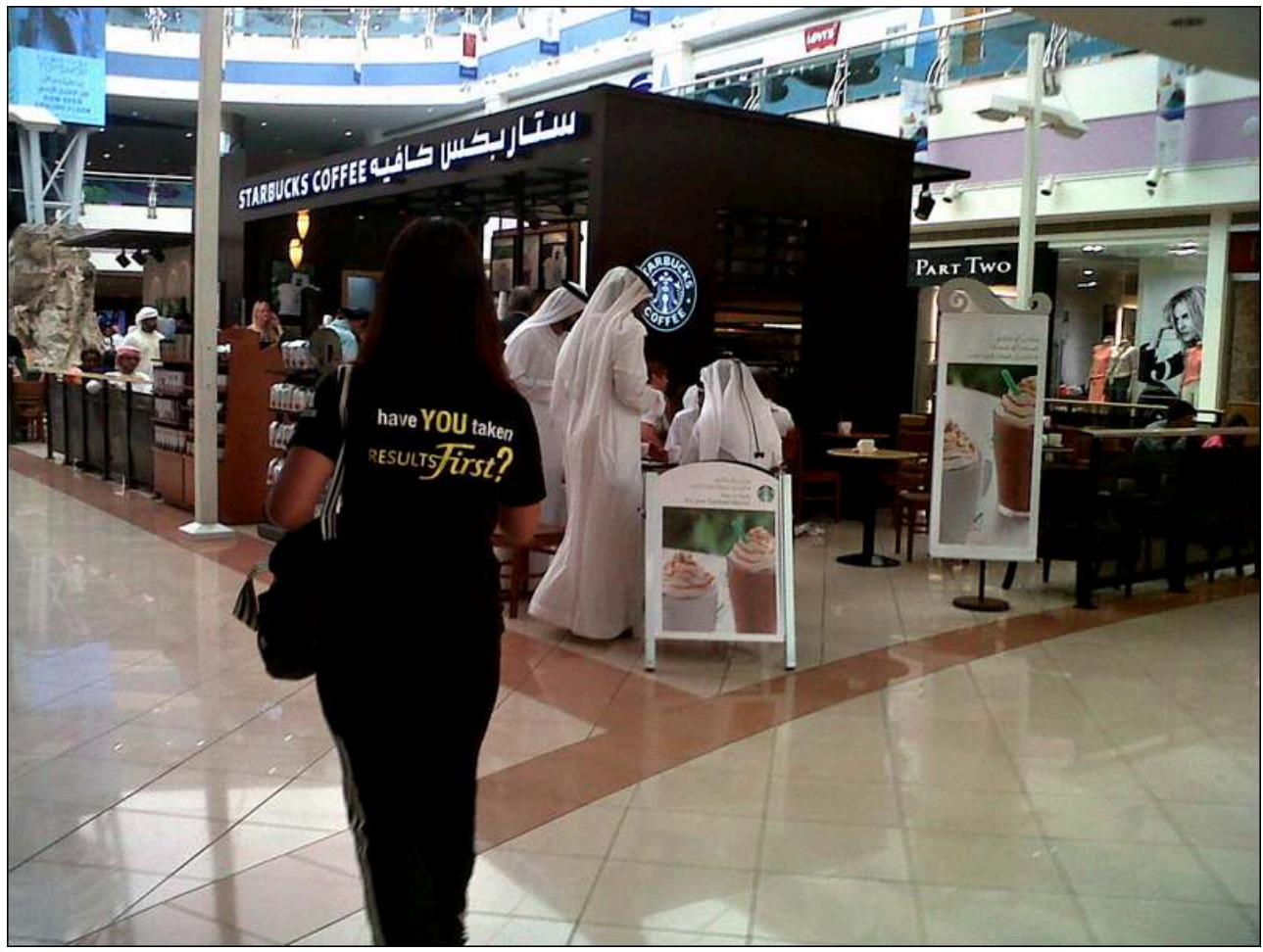

21 Les malls et les cafés internationaux sont par conséquent des lieux importants de rencontres, et parfois de drague, à distance, par l'intermédiaire de numéros de téléphone transmis sur des bouts de papier ou, plus récemment, des messageries 
instantanées des téléphones portables connectés à internet ou au réseau bluetooth. Ils sont aussi les lieux d'une sociabilité de couple, où se rendent à la fois les jeunes couples récemment mariés, et les couples non mariés mais qui considèrent leur relation comme sérieuse. Les jeunes gens que j'ai rencontrés tendaient en effet à catégoriser les relations amoureuses entre les relations de flirt, de rendez-vous amoureux (dating), considérées comme peu durables, et les relations sérieuses, qui ont pour suite logique le mariage. Ces dernières vont souvent de pair avec une certaine retenue dans les marques d'affection, et notamment l'abstinence sexuelle, considérée comme un gage de sérieux et un engagement de la part du jeune homme, puisqu'il contribue à préserver la réputation de sa future épouse ${ }^{17}$. L'évolution typique de ce type de relations serait qu'après un certain nombre de rencontres, la jeune femme en parle à sa mère, qui se fait l'intermédiaire auprès du père et, lorsque ce dernier a donné son assentiment, le jeune homme vient faire la demande en mariage.

L'un des enjeux du secret gardé sur les relations amoureuses peut être, à l'inverse, la revendication $\mathrm{du}$ caractère précaire, flexible, transitoire de ces relations - par opposition au mariage, seul horizon légitime (Kreil, 2012). L'accent est alors mis sur le jeu, l'expérimentation, et les lieux choisis sont ces espaces résiduels de la planification urbaine où il est plus aisé de se dissimuler et où les normes habituelles peuvent être provisoirement levées.

Mahmoud, un Égyptien qui a grandi à Abou Dhabi, se rappelle son premier amour : il l'a rencontrée l'été de ses quatorze ans, au Liwa Centre (centre commercial au centre-ville d'Abou Dhabi, qui a précédé la création des grands shopping malls), où ses amis et lui passaient le plus clair de leur temps à marcher dans les allées. Leur premier baiser a eu lieu dans une villa abandonnée du quartier de Khalidiya (illustration 3), où quelques groupes d'adolescents avaient l'habitude de se retrouver pour boire de l'alcool en cachette - la villa a depuis été détruite. La plage de Ra's Al-Akhdâr, disparue aujourd'hui elle aussi, fait également partie de ces interstices cités avec nostalgie par mes interlocuteurs, et qui ont souvent été les territoires de leurs initiations amoureuses. 
Illustration 3 - Villas et parkings à l'intérieur d'un bloc d'immeubles (Khalidiya, Abou Dhabi)

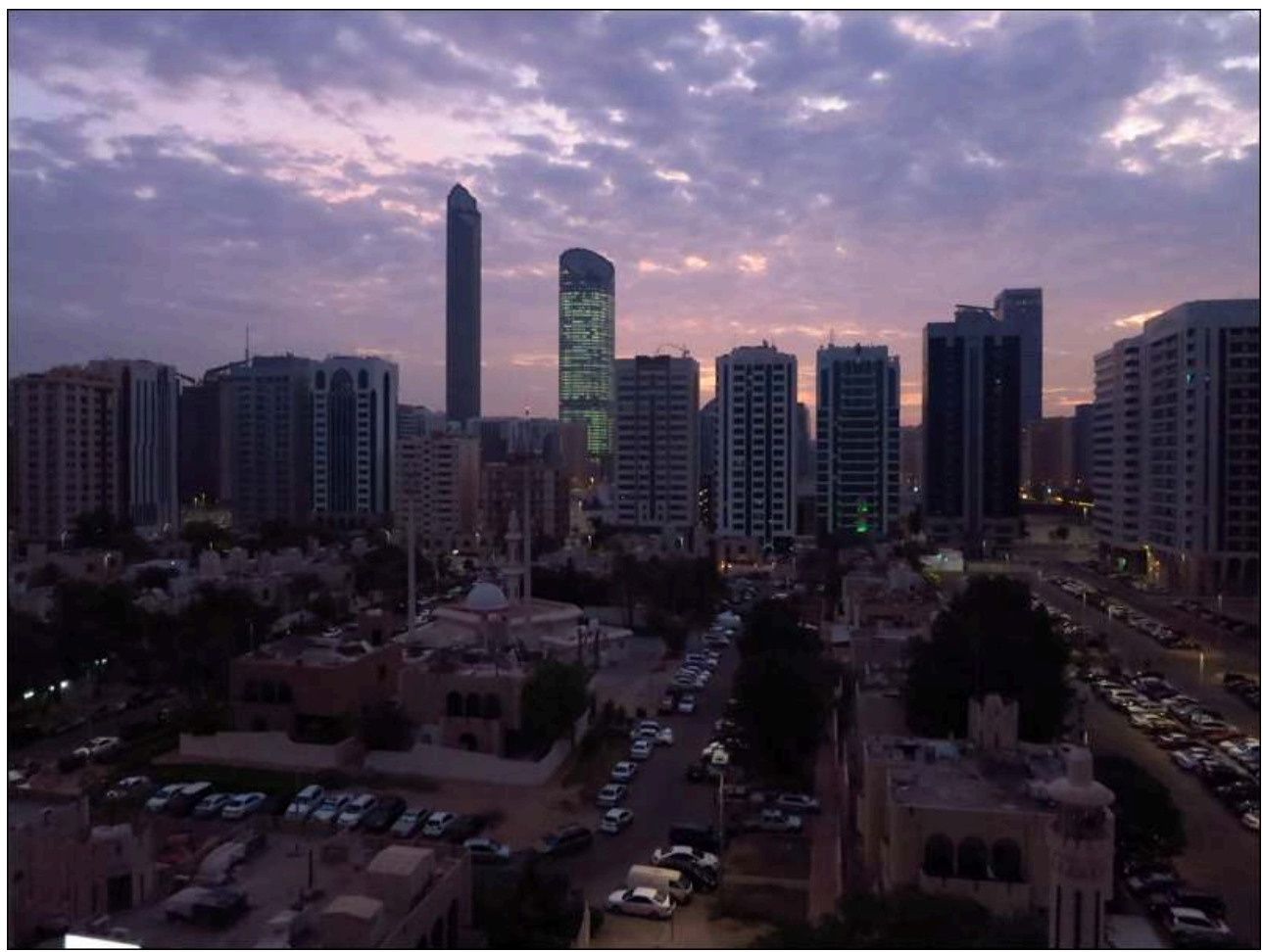

24 L'un des équivalents de ces lieux aujourd'hui pourrait être la jetée située à l'ouest d'Abou Dhabi, qui se prolonge le long du Heritage Village et derrière le théâtre national. Durant la journée, des femmes de tous âges viennent s'y promener, souvent accompagnées d'enfants en poussettes, et y admirer la vue sur la corniche d'Abou Dhabi et les rangées d'immeubles qui la bordent. Le soir et jusque très tard dans la nuit, la jetée devient un espace de jeux, de rencontres et de drague pour les jeunes adultes, en majorité émiriens et expatriés arabes. La plupart d'entre eux s'y rendent en voiture, et les numéros de téléphone s'échangent à travers les vitres teintées entrouvertes, desquelles s'échappe de la musique à fort volume. D'autres s'installent en groupes homosociaux sur les parkings, pour discuter ou écouter de la musique en comparant les qualités respectives de leurs voitures souvent personnalisées. Certains groupes s'assoient au bout de la jetée pour pique-niquer face à la corniche d'Abou Dhabi; de jeunes couples se promènent sur la plage (illustration 4). Ces derniers sont néanmoins sous haute surveillance: du crépuscule à l'aube, une voiture de police se trouve constamment derrière le théâtre, sans doute en raison des rumeurs de prostitution qui courent parfois sur cet endroit. 
Illustration 4 - La plage derrière le théâtre national, au bout de la jetée, en fin d'après-midi (Abou Dhabi)

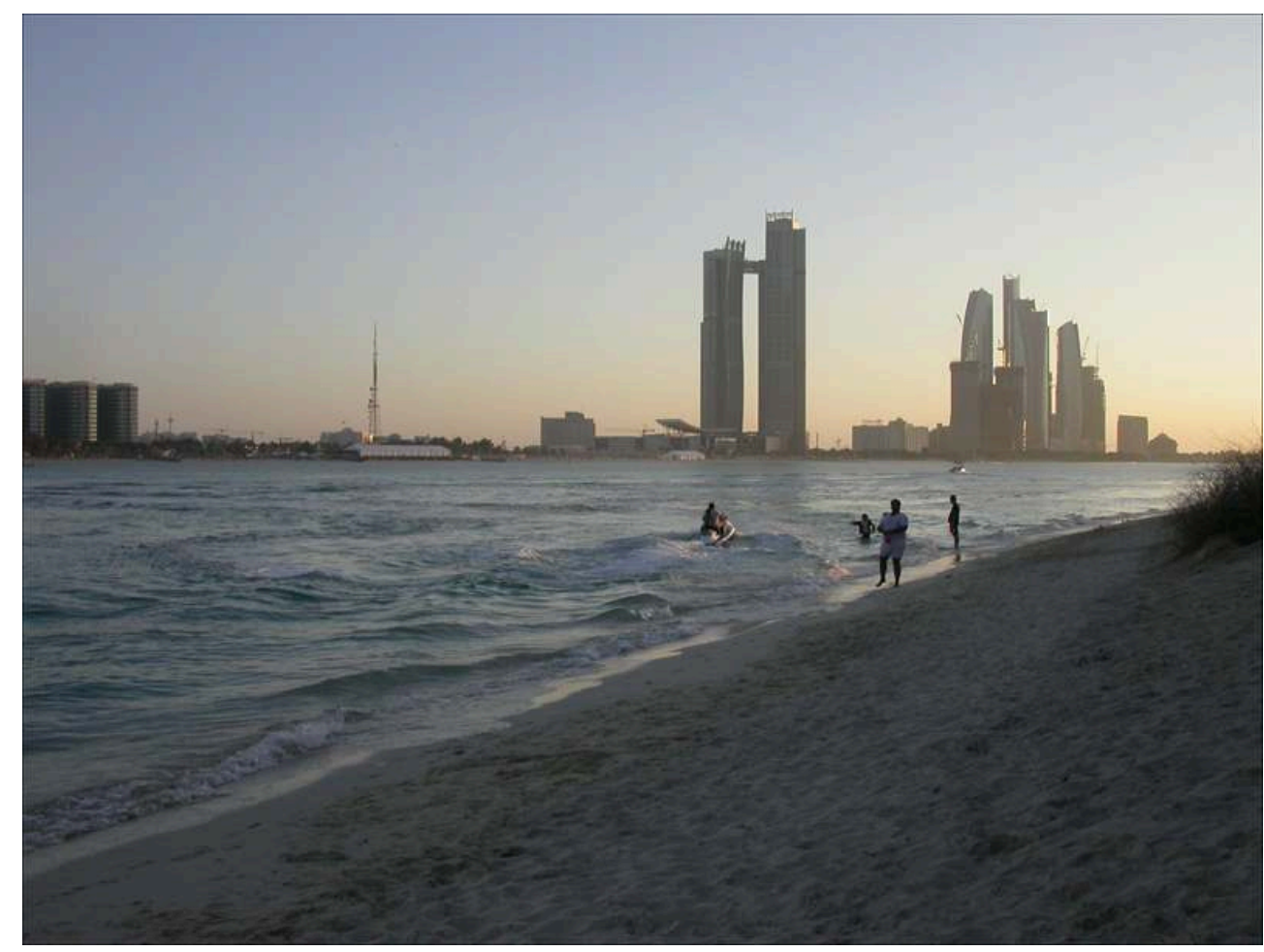

\section{Les rencontres amoureuses et l'ailleurs}

L'exemple de la jetée, comme les précédents, illustre l'importance de la voiture dans ces sociabilités amoureuses. Instrument de mobilité qui permet de se déplacer dans les villes émiriennes très étendues et où les transports publics sont peu développés, la voiture est aussi un outil d'autonomie pour les jeunes adultes. Elle permet de se déplacer loin de l'environnement immédiat - et donc des lieux où il est le plus probable de rencontrer des connaissances - et fournit en même temps un espace d'invisibilité relative. Ahmad, le jeune Syrien déjà évoqué, raconte par exemple comment il a contacté, sur le réseau social Facebook, une étudiante émirienne qui fréquentait le campus féminin de la même université. Après avoir discuté quelque temps en ligne, puis par la messagerie instantanée de leurs téléphones (qui fonctionne par code pin et permet donc de conserver l'anonymat), ils passent à la conversation téléphonique. Un jour, alors qu'ils sont tous les deux à l'université, avec une longue pause entre deux cours, elle lui propose de venir le chercher en voiture; lui-même n'en possède pas, et vient à l'université en bus. La jeune femme passe donc le prendre à l'entrée du campus masculin, et conduit jusqu'à l'autre bout de la ville. Elle se gare dans un parking un peu à l'écart. Sans sortir de la voiture, ils discutent durant une heure, puis retournent vers leurs campus respectifs pour assister à leurs derniers cours de la journée.

L'invisibilité et la mobilité offertes par la voiture permettent ici la rencontre physique. Cette anecdote est intéressante à bien d'autres niveaux : les hiérarchies traditionnelles de genre sont renversées au profit d'une hiérarchie de statut économique. C'est la jeune femme qui possède le véhicule, conduit, et prend l'initiative de la rencontre. Par ailleurs, cet exemple montre comment les relations sont inclues dans une hiérarchie 
d'étapes, depuis la discussion en ligne jusqu'à la rencontre physique en passant par la conversation téléphonique, qui naviguent entre des degrés différents et flexibles de distance et d'intimité. L'articulation entre les deux est complexe: la rencontre « virtuelle ", qui peut sembler plus sûre en raison du degré plus grand d'anonymat, se fait cependant à partir du point commun de l'université, ce qui remet en question l'idée des relations en ligne comme délocalisées ou déconnectées de l'identité réelle de leurs usagers (Miller, Slater, 2000 ; Delpal, 2003). Internet apparait ici comme un outil permettant de nouer des relations en contournant les contraintes qui pèsent sur les rencontres entre jeunes hommes et femmes dans les espaces publics (Le Renard, 2011a) : s'il est virtuel, c'est au sens de la potentialité, des rencontres offertes qui s'actualisent ou non dans l'espace physique. Il est aussi un moyen de prolonger ces relations jusque dans l'espace domestique, à travers les systèmes de messageries instantanées, tout en assurant une certaine confidentialité.

De la même manière, l'espace public à Abou Dhabi est loin d'être anonyme, puisqu'il est nécessaire de rester dans la voiture par peur d'être reconnu. L'anonymat nécessaire aux relations amoureuses passe ainsi très souvent par le déplacement des rencontres au-delà des frontières de la ville. Pour les jeunes adultes d'Abou Dhabi, les restaurants et les cafés de Dubaï constituent souvent des lieux plus anonymes, car fréquentés par une foule plus nombreuse, et plus éloignés des espaces familiers où ils risquent de croiser des connaissances ou des membres de la famille élargie. Il n'est pas rare que les jeunes couples fassent séparément le trajet jusqu'au lieu de rendez-vous à Dubaï, dînent ensemble, puis repartent chacun de leur côté vers Abou Dhabi. Cet espace public des rencontres est parfois étendu à l'étranger ${ }^{18}$, comme en témoigne un courant récent de blogs sur lesquels sont publiées sous forme de séries des histoires d'amour fictives. Lancés au Koweït aux environs de 2008, ces blogs se sont ensuite propagés dans l'ensemble de la région, du Bahreïn aux Émirats, et connaissent un certain succès. Ils sont écrits majoritairement par des jeunes femmes du Golfe, en arabe translittéré et en anglais, et combinent des histoires d'amour romantique hétéronormatives, proches de celles des séries télévisées étrangères très populaires (dernièrement, les séries turques et coréennes), tout en respectant les normes sociales propres aux pays du Golfe. Or, la grande majorité de ces récits commencent par une rencontre à l'étranger - New York, Londres ou ailleurs, au hasard d'un aéroport, des cafés internationaux ou des couloirs d'un grand hôtel où les protagonistes passent leurs vacances en famille. Les scènes de rencontre et de séduction jouent en général sur l'incertitude quant à la nationalité de l'interlocuteur, et donnent lieu à des scènes cocasses lorsque les deux jeunes gens réalisent avec stupéfaction qu'ils sont concitoyens. Ces histoires jouent à la fois sur un imaginaire de l'ailleurs transgressif, et sur la réalité des vacances à l'étranger des familles du Golfe, qui bien souvent se rendent dans les mêmes lieux et y reproduisent les pratiques habituelles (Beaugrand, 2013).

\section{Conclusion}

L'examen des espaces vécus et imaginés des rencontres amoureuses aux Émirats arabes unis permet d'aborder la question des normes sociales, et en particulier de l'inscription des hiérarchies ethniques et de genre dans l'espace : les valeurs sociales attribuées aux lieux semblent en effet cristalliser des enjeux propres à la composition et aux évolutions de la société émirienne. Surtout, ces espaces posent la question de la 
transgression, qu'elle soit vécue ou rêvée, et l'on peut s'interroger sur sa portée : à quel point les pratiques et les récits évoqués sont-ils porteurs de changement, et contribuent-ils eux-mêmes à déplacer les normes?

Il semble bien plutôt que les pratiques évoquées dans ce texte correspondent à un moment dans le cycle de vie de ces jeunes adultes ${ }^{19}$, et s'inscrivent au sein de sociabilités quotidiennes propres à cette génération urbaine. En ce sens, les relations évoquées feraient figure de jeu toléré et provisoire avec les normes, qui se résoudrait dans le mariage - soit qu'il constitue l'issue de la relation amoureuse, soit que ces pratiques soient abandonnées à son profit. Ces transgressions respectables s'opposent aux pratiques officiellement identifiées comme déviantes et qui font l'objet de traitements institutionnels, psychiatriques ou judiciaires, comme c'est le cas pour l'homosexualité (Al-Qasimi, 2012 ; Le Renard, 2011a).

Cela ne signifie pas pour autant que les pratiques et les récits mentionnés sont sans effet. Si le mariage constitue un moment de choix, où les normes sociales sont réaffirmées ou brisées, il existe de multiples stratégies pour retarder ou contourner, précisément, ce choix - en poursuivant des études longues par exemple. De même, le cosmopolitisme réel mais peu accessible des villes émiriennes semble être transféré dans le domaine de l'imaginaire, où les récits mobilisent des références culturelles multiples pour véhiculer les aspirations d'une génération. Ainsi, si les héroïnes d'histoires d'amour publiées sur les blogs finissent toujours, classiquement, par épouser le héros, elles revendiquent au passage leur droit à choisir leur filière d'études et à prendre les décisions concernant leur avenir, ou encore mettent en avant une vision du couple où homme et femme collaborent à rôles égaux.

\section{BIBLIOGRAPHIE}

Al-Qasimi N., 2010. Ladies and Gentlemen, Boyahs and Girls: Uploading Transnational Queer Subjectivities in the United Arab Emirates. In Hegde R. (dir.), Circuits of Visibility: Gender and Transnational Media, New York, New York University Press.

Al-Qasimi N., 2012. The "Boyah" and the "Baby Lady": Queer Mediations in Fatima Al Qadiri and Khalid Al Gharaballi's Wawa Series (2011). Journal of Middle East Women's Studies, 8, n³, p. 139-142.

Assaf L., 2013. Autour d'un café. Sociabilité des jeunes à Abu Dhabi. In Bonnefoy L., Catusse M., Jeunesses arabes. Du Maroc au Yémen : loisirs, cultures et politiques, Paris, La Découverte, p. 53-62.

Banos V., 2009. Introduction : de l'espace à la dimension spatiale des normes sociales. Géographie et cultures [En ligne], 72 | 2009, mis en ligne le 06 mai 2013, consulté le 14 août 2013. URL : http:// gc.revues.org/ 2183

Beaugrand C., 2013. Les vacances en Europe. Univers familial, univers familier des « Golfiens » ». In Bonnefoy L., Catusse M., Jeunesses arabes. Du Maroc au Yémen : loisirs, cultures et politiques, Paris, La Découverte, p. 225-229. 
Bristol-Rhys J., 2007. Weddings, Marriage and Money in the United Arab Emirates. Anthropology of the Middle-East, vol. 2, $\mathrm{n}^{\circ}$ 1, p. 20-36.

Bristol-Rhys J., 2010. Emirati Women. Generations of Change. London, Hurst \& Company.

Capron G., 2000. Rassemblement et dispersion dans la ville latino-américaine : un nouvel espace public, le cas du centre commercial. Cahiers des Amériques latines, vol. 35, n 3, p. 21-40.

Davidson C., 2009. Abu Dhabi: Oil and Beyond. New York, Columbia University Press.

De Koning A., 2009. Gobal Dreams: Class, Gender and Public Space in Cosmopolitan Cairo. Cairo, The American University in Cairo Press.

Delpal C., 2003. Tisser la toile à son image : Les cybercafés de Beyrouth. In Mermier F. (dir.), Mondialisation et nouveaux médias dans l'espace arabe, Paris, Maisonneuve \& Larose, p. 205-231.

Dresch P., 2005. Debates on Marriage and Nationality in the United Arab Emirates. In Dresch P., Piscatori J. (ed.), Monarchies and Nations. Globalisation and Identity in the Arab States of the Gulf, Londres, I. B. Tauris, p. 136-157.

Elsheshtawy Y., 2010. Dubai: Behind an Urban Spectacle. Londres, Routledge.

Fuccaro N., 2009. Histories of City and State in the Persian Gulf. Cambridge, Cambridge University Press.

Gardner A., 2013 (à paraître). The Amalgamated City: Petroleum Wealth and Urban Space in Doha, Qatar. Arabian Humanities, $\mathrm{n}^{\circ} 2$.

Gillot G., 2005. Faire sans le dire. Les rencontres amoureuses au Caire. Géographie et cultures, $\mathrm{n}^{\circ} 54$, p. 31-52.

Goffman E., 1973. La mise en scène de la vie quotidienne. Paris, Minuit.

Hastings M., Nicolas L., Passard C., 2012. Paradoxes de la transgression. Editions La Martinière.

Heard-Bey F., 2005. From Trucial States to United Arab Emirates. Dubai, Motivate Publishing.

Johnson-Hank J., 2002. On the Limits of Life Stages in Ethnography: Toward a Theory of Vital Conjunctures. American Anthropologist, Vol. 104, No. 3, p. 865-880.

Kanna A., 2011. Dubai, the City as Corporation. Minneapolis, University Of Minnesota Press.

Kapiszewski A., 2001. Nationals and Expatriates: Population and Labour Dilemmas of the Gulf Cooperation Council States. New York, Ithaca Press.

Khalaf S., 2005.National dress and the construction of Emirati cultural identity. Journal of Human Sciences, $\mathrm{n}^{\circ} 11$.

Kreil A., 2012. Du rapport au dire. Sexe, amour et discours d'expertise au Caire. Thèse en Anthropologie sociale et ethnologie, Paris, EHESS.

Lagrange F., 2008. Islam d'interdits, islam de jouissance. Paris, Téraèdre.

Lavergne M., 2005. Sur les ailes du désir, de Koweït à Mascate. Villes et territoires du Moyen-Orient, 1, http://halshs.archives-ouvertes.fr/docs/00/37/51/52/PDF/desir_lavergne.pdf

Le Renard A., 2011a. Femmes et espaces publics en Arabie Saoudite. Paris, Dalloz.

Le Renard A., 2011b. Genre, classe, nationalité et accès des femmes aux espaces publics à Riyad. Sociétés contemporaines, $\mathrm{n}^{\circ} 84$, p. 151-172. 
Le Renard A., 2013. Les buya. Subversion des normes de genre en Arabie Saoudite. In Bonnefoy L., Catusse M., Jeunesses arabes. Du Maroc au Yémen : loisirs, cultures et politiques, Paris, La Découverte, p. 68-77.

Longva A. N., 1997. Walls Built on Sand: Migration, Exclusion and Society in Kuwait. Boulder, Westview Press.

Longva A. N., 1999. Keeping Migrant Workers in Check. The Kafala System in the Gulf. Middle East Report, 211.

Miller D., Slater D., 2000. The Internet: An Ethnographic Approach. Oxford, Berg.

Moghadam A., 2013 (à paraître). De l'Iran imaginé aux nouveaux foyers de l'Iran : Pratiques et espaces transnationaux des Iraniens à Dubaï. Arabian Humanities, $\mathrm{n}^{\circ} 2$.

Naciri M., 1997. Le rôle de la citadinité dans l'évolution des villes arabo-islamiques. In Naciri M., Raymond A. (dir.), Sciences sociales et phénomène urbain dans le monde arabe, Casablanca, Fondation du Roi Abdul-Aziz Al Saoud pour les Études Islamiques et les Sciences Humaines, p. 131-148.

Olivier de Sardan J.-P., 1995. La politique du terrain. Enquête, n¹, En ligne, mis en ligne le 10 juillet 2013, consulté le 17 septembre 2013. URL : http://enquete.revues.org/263

Schwartz O., 1993. L'empirisme irréductible. Postface à Le hobo : sociologie du sans-abri, Anderson N., Nathan, p. 265-306.

Statistics Center - Abu Dhabi, 2011. Statistical Yearbook of Abu Dhabi 2011.

\section{NOTES}

1. La plage de Ra's Al-Akhdâr était située à l'extrémité nord-ouest d'Abou Dhabi. Elle a été fermée aux alentours de 2010 pour étendre l'hôtel Emirates Palace et construire le nouveau palais présidentiel, mais un certain nombre de mes interlocuteurs voyaient cette fermeture comme une tentative de contrôler les sociabilités amoureuses qui faisaient la renommée du lieu. Le parking de l'hôtel Intercontinental est situé un peu en périphérie du centre-ville d'Abou Dhabi, vers l'ouest; Officers' Club est lui aussi à l'écart, au sud de l'île. À l'inverse, Al-Wahda Mall est au centre-ville ; il comporte un très grand parking, sur plusieurs étages, dans un immeuble adjacent au shopping mall.

2. L'importance de ces changements est néanmoins à nuancer dans le cas des villes portuaires dont le développement précède la découverte du pétrole, comme c'est le cas de Dubaï, à l'instar d'autres villes du Golfe (Fuccaro, 2009).

3. L'étymologie du terme «transgresser » signifie traverser, passer outre, franchir une limite (Hastings et al., 2012).

4. Les entretiens cités dans cet article se sont déroulés majoritairement en anglais, mêlé parfois d'arabe - le mélange des deux langues est caractéristique du langage des jeunes à Abou Dhabi. Certains termes, qui renvoient à des pratiques et des notions culturellement spécifiques, étaient utilisés exclusivement dans l'une des deux langues par mes interlocuteurs. Ils apparaissent dans ce texte entre parenthèses après leur traduction en français.

5. Plus souvent que harâm (l'interdit religieux), le terme employé est 'ayb, l'interdit social, ce qui est aussi une manière de s'en distancier, de l'atténuer - les normes sociales pouvant être critiquées et modifiées.

6. Lors d'un entretien réalisé en mars 2010.

7. Il existe une importante littérature anthropologique sur la question de la réputation dans le monde arabe et de son lien avec la catégorie de l'honneur (Abu-Lughod, 2009). A l'instar d'Amélie 
Le Renard (2011a), la réputation est ici envisagée comme catégorie indigène, et en termes de ses conséquences pratiques sur l'accès aux espaces publics des jeunes femmes.

8. Il faut préciser ici que j'avais rencontré les deux étudiantes dans leur université, où l'autorisation de mener des entretiens venait de m'être refusée. C'est l'une des deux jeunes femmes, Shayma, qui a proposé le lieu et nous y a conduites dans sa voiture depuis l'université.

9. Il n'existe pas de statistiques fiables ou officielles de la répartition par nationalité de la population étrangère, même si le sujet est souvent évoqué dans la presse. Les Indiens représenteraient $30 \%$ de la population, les Pakistanais et Bangladais $28 \%$ environ. Après eux viennent les citoyens d'autres pays arabes, les Iraniens, les Philippins et les citoyens d'autres pays asiatiques (environ $17 \%$ ) et les expatriés occidentaux (environ $8 \%$ ). Ces chiffres varient suivant les émirats : par exemple, les Iraniens sont majoritairement installés à Dubaï (Moghadam, 2013). Les spécialisations professionnelles et les niveaux de salaire recoupent souvent ces catégories ethniques, aux Émirats arabes unis comme dans le reste du Golfe (Longva, 1997).

10. Etre citoyen des Émirats arabes unis se traduit par un accès gratuit à l'éducation et à la santé, et des subventions importantes pour le logement et le mariage (lorsqu'il a lieu avec un autre citoyen).

11. Comme en témoignent par exemple les diverses campagnes lancées par des femmes émiriennes sur internet pour faire respecter les codes vestimentaires dans les shopping malls, qui visent les vêtements trop courts portés par les femmes occidentales ou asiatiques. Il faut noter que la population nationale est loin de soutenir unanimement ces campagnes, qui ont fait l'objet de controverses virulentes sur les réseaux sociaux et dans les médias nationaux.

12. Le système éducatif est un bon exemple de ces pratiques de distinction - et des contradictions qu'elles engendrent. Dans les Émirats, les écoles et universités publiques, majoritairement réservées aux nationaux (même si elles sont fréquentées par une petite proportion d'étudiants étrangers), sont ségrégées en fonction du genre; ce qui n'est pas le cas des écoles privées, qui suivent pour la plupart des cursus étrangers, et sont considérées comme plus prestigieuses. Le choix d'une filière éducative pour les jeunes femmes balance donc souvent entre le caractère valorisé de l'éducation dans une université étrangère, et le maintien de la ségrégation entre les sexes. Dans la ville d'Abou Dhabi en 2009-2010, $68 \%$ des élèves nationaux et $19 \%$ des élèves étrangers étaient scolarisés dans le secteur public, du 1er cycle au secondaire (Statistics Center Abu Dhabi, 2011).

13. La 'abâya est une longue robe noire portée par-dessus les vêtements par les femmes du Golfe. Il en existe différentes sortes; dans les Émirats, elle se porte sur les épaules et est souvent ouverte en bas. Elle est souvent associée à la shayla, voile noir léger posé sur la tête.

14. Une bonne illustration de cette nécessité est l'interdiction (pas toujours appliquée) de servir de l'alcool à une personne portant l'habit national, qui oblige en théorie les Émiriens à porter d'autres vêtements s'ils veulent se rendre dans un bar.

15. Les quatre histoires, dont les scènes sont alternées dans le film, ont été ici distinguées pour plus de clarté, et ne font donc pas justice aux choix de montage et au suspense maintenu durant le film.

16. L'idée que le caractère trop strict des interdits encourage les déviances est assez répandue parmi les jeunes gens que j'ai interrogés. On la retrouve aussi dans d'autres courts-métrages, par exemple « 3aib» (2012), dont la réalisatrice est restée anonyme. Le film raconte l'histoire d'une relation entre la narratrice et un homme marié, père de deux enfants. La cause de cette transgression est attribuée à la catégorie de 'ayb (ce qui est honteux, réprouvé) : puisque tout est 'ayb dans les relations entre hommes et femmes, il deviendrait impossible de distinguer les petites transgressions des grandes, et le bien du mal.

17. Beaucoup de jeunes femmes expriment la crainte que les promesses de mariage ne soient destinées qu'à obtenir des faveurs sexuelles, et de nombreux récits circulent sur des femmes abandonnées par un amant qui, après avoir consommé l'acte, refuse de les épouser parce que leur 
réputation est salie. On trouve l'un de ces récits dans le roman saoudien Les filles de Riyad, de Rajaa Alsanea (Paris, Plon, 2007). Ces récits assurent une certaine fonction sociale normative, dans la mesure où ils contribuent à dissuader les jeunes femmes d'avoir des relations sexuelles avant le mariage.

18. Cette idée rejoint une proposition de Mohamed Naciri (1997), pour qui la citadinité des villes du Golfe se situe à l'étranger, dans un espace public étendu à Londres, Paris ou New York.

19. Il ne s'agit pas ici de considérer le cycle de vie comme un schéma fixe d'étapes suivies linéairement, mais plutôt comme un ensemble de « conjonctures vitales » (Johnson-Hank, 2002), c'est-à-dire de configurations construites socialement autour d'événements vitaux (le mariage, la naissance, le travail, etc.) dont l'ordre et surtout la synchronisation varient. Ainsi les pratiques décrites dans ce texte concernent à la fois des étudiants et des jeunes professionnels : dans le contexte émirien, le mariage semble constituer une conjoncture vitale bien plus significative socialement que l'entrée dans le monde du travail, même si cette dernière agit, comme on l'a vu, sur les possibilités de mixité et d'interactions entre les genres.

\section{RÉSUMÉS}

Les relations amoureuses des jeunes adultes dans les Émirats arabes unis, qu'elles soient vécues ou fictives, prennent souvent une dimension spatiale importante. L'espace récent des villes émiriennes cristallise en effet des normes sociales renforcées par la cohabitation de populations variées. Des parkings aux shopping malls, les jeunes adultes mobilisent les possibilités des lieux pour s'émanciper provisoirement de ces normes, en jouant sur la frontière entre le transgressif et le respectable, mais aussi sur un ailleurs qui peut être celui des espaces imaginés dans les productions culturelles, blogs et courts-métrages, ou encore celui d'internet et des réseaux sociaux en ligne.

The love relationships of young adults in the United Arab Emirates often take on an important spatial dimension, whether they are lived or fictional. The recent urban space of Emirati cities crystallizes indeed social norms that are reinforced by the coexistence of varied populations. From the parking lots to the shopping malls, young adults use the potentialities of space to emancipate themselves, although temporarily, from these norms, while playing on the boundary between transgression and respectability, but also while looking for other horizons in the imagined spaces of their cultural productions, blogs and short movies, or within online social networks.

\section{INDEX}

Mots-clés : Émirats arabes unis, jeunes, relations amoureuses, espace public, transgression

Keywords : United Arab Emirates, youth, love relationships, public space, transgression

Thèmes : Sur le Champ - Sur le Terrain 
AUTEUR

\section{LAURE ASSAF}

Laure Assaf, laure.assaf@gmail.com, est Doctorante contractuelle à l'Université Paris Ouest Nanterre La Défense, Laboratoire d'ethnologie et de sociologie comparative. 